\title{
Impacto econômico da retenção de placenta em vacas leiteiras ${ }^{1}$
}

\author{
Myriam M. Nobre ${ }^{2}$, Rafael A. Azevedo ${ }^{2 *}$, Ernane F. Campos ${ }^{3}$, Camila F.A. Lage ${ }^{2}$, \\ Joana R. Glória², Helton M. Saturnino² e Sandra G. Coelho²
}

\begin{abstract}
Nobre M.M., Azevedo R.A., Campos E.F., Lage C.F.A., Glória J.R., Saturnino H.M. \& Coelho S.G. 2018. [Economic impact of retained placenta in dairy cows.] Impacto econômico da retenção de placenta em vacas leiteiras. Pesquisa Veterinária Brasileira 38(3):450-455. Departamento de Zootecnia, Escola de Veterinária, Universidade Federal de Minas Gerais, Avenida Antônio Carlos 6627, Belo Horizonte, MG 30123-970, Brazil. E-mail: rafaelzooufmg@gmail.com

Approximately $75 \%$ of diseases in dairy cows occur in the first month after parturition and these problems have their origin associated with the immune system and food consumption two to three weeks before parturition. Diseases related to the reproductive tract, such as retained placenta, can affect reproductive efficiency as well as milk production. The effects of diseases on the physiological processes of animals become economic impacts that can be measured. The objective of this study was to evaluate the economic impact of retained placenta on a herd of 900 lactating cows. The diagnosis of placental retention was defined as presence of the placenta 24 hours postpartum. A database was used in the study, from which the following information was extracted: placenta presence 24 hours after calving, year of calving, calving season, and number of semen doses per gestation. In order to calculate the economic impact, the direct costs (treatment, labor, reduction of milk production and milk discharge during the treatment period) and indirect costs (increase in the service period, increase in the number of semen doses and increase of the risk of disposal). The average values related to the costs were obtained from the veterinarian responsible for the property with reference to the year 2009 for the calculations to be carried out. The total cost per occurrence of placenta retention in primiparous cows in the rainy and dry season was US\$51.8 and in multiparous cows was US\$70.6 and US\$87.9 in times of drought and rain, respectively. Retention of placenta presented a cost for the property in the evaluated period of US\$8,878.0 or 19,666 liters of milk. Retention of placenta presented a significant economic impact on milk production under the conditions evaluated.

INDEX TERMS: Economic impact, retained placenta, dairy cows, cattle, costs, reproductive disease, milk production, clinics.
\end{abstract}

RESUMO.- Aproximadamente $75 \%$ das doenças em vacas leiteiras acontecem no primeiro mês após o parto e esses problemas têm sua origem associada ao sistema imune e ao consumo de alimentos duas a três semanas antes do parto. Doenças relacionadas ao trato reprodutivo como, por exemplo,

\footnotetext{
${ }^{1}$ Recebido em 21 de maio de 2017.

Aceito para publicação em 22 de junho de 2017.

2 Departamento de Zootecnia, Escola de Veterinária, Universidade Federal de Minas Gerais (UFMG), Avenida Antônio Carlos 6627, Belo Horizonte, MG 30123-970, Brasil. *Autor para correspondência: rafaelzooufmg@gmail.com

${ }^{3}$ Recursos Humanos no Agronegócio (ReHagro), Avenida Uruguai 620, Sion, Belo Horizonte, MG 30310-300, Brasil.
}

retenção de placenta pode afetar a eficiência reprodutiva assim como a produção de leite. Os efeitos das doenças nos processos fisiológicos dos animais se transformam em impactos econômicos passíveis de serem mensurados. Objetivou-se avaliar o impacto econômico da retenção de placenta em um rebanho composto de 900 vacas em lactação. O diagnóstico de retenção de placenta foi definido como presença da placenta 24 horas após o parto. Foi utilizado um banco de dados no estudo, do qual foram extraídas as seguintes informações: ano de parição, época de parição, ordem de lactação, presença da placenta 24 horas após o parto, e número de doses de sêmen por gestação. Para cálculo do impacto econômico foram considerados os custos 
diretos (tratamento, mão de obra, redução da produção de leite e descarte de leite durante o período de tratamento) e indiretos (aumento do período de serviço, aumento do número de doses de sêmen e aumento do risco de descarte). Os valores médios relacionados aos custos foram obtidos junto ao veterinário responsável pela propriedade com referência ao ano de 2009 para que os cálculos fossem realizados. 0 custo total por ocorrência de retenção de placenta em vacas primíparas no período de chuva e seca foi de US $\$ 51,8$ e em vacas multíparas foi de US\$70,6 e US\$87,9 nas épocas de seca e de chuvas, respectivamente. A retenção de placenta apresentou custo para a propriedade no período avaliado de US $\$ 8.878,0$ ou 19.666 litros de leite. A retenção de placenta apresentou impacto econômico importante na propriedade leiteira nas condições avaliadas.

TERMOS PARA INDEXAÇÃO: Impacto econômico, placenta, vacas leiteiras, bovinos, custos, doença reprodutiva, produção de leite, clínica.

\section{INTRODUÇÃO}

A definição de retenção de placenta é baseada no processo fisiológico de expulsão da membrana fetal do útero de vaca (Zobel \& Tkalčić 2013) e se caracteriza como a falha da expulsão da placenta dentro de 12 a 24 horas após o parto (Kelton et al. 1998, Fourichon et al. 1999), representando condição patológica (Zobel \& Tkalčić 2013). A liberação da placenta envolve a perda da adesão materno-fetal e ocorre somente após a maturação do placentoma, sendo que as contrações uterinas ajudam mecanicamente essa liberação que ocorre fisiologicamente entre três e seis horas após o parto (Meça et al. 2006). Os processos que conduzem essa separação e a liberação começam antes do parto e envolvem o sistema imune (Beagley et al. 2010).

A ocorrência de doença pode resultar em mortalidade, redução da produção de leite, redução da fertilidade e descarte (Fourichon et al. 1999). Segundo LeBlanc et al. (2006), aproximadamente $75 \%$ das doenças em vacas leiteiras acontecem no primeiro mês após o parto e esses problemas têm sua origem associada ao sistema imune e ao consumo de alimentos duas a três semanas antes do parto. Tem sido demonstrado que doenças relacionadas ao trato reprodutivo como, por exemplo, a retenção de placenta, podem afetar a eficiência reprodutiva assim como a produção de leite (Rajala \& Grohn 1998, Fourichon et al. 1999, Han \& Kim 2005), além de gerar problemas financeiros a bovinocultura (Farzaneh et al. 2006).

Segundo Dijkhuizen \& Morris (1996), os efeitos das doenças nos processos fisiológicos dos animais se transformam em impactos econômicos passíveis de serem mensurados. Para se quantificar as perdas econômicas, é necessário utilizar modelos de simulação das consequências da doença em um rebanho.

Dessa maneira, torna-se importante o desenvolvimento de metodologias de trabalho que determinem o impacto econômico das doenças no sistema de produção assim como a difusão desse conhecimento visando sensibilizar os gestores das propriedades leiteiras para o planejamento e a implementação de medidas preventivas eficazes. Objetivou-se com esse trabalho avaliar o impacto econômico da retenção de placenta em uma propriedade leiteira.

\section{MATERIAL E MÉTODOS}

Avaliou-se o banco de dados de uma fazenda localizada no estado de Minas Gerais, contendo informações de partos ocorridos de janeiro de 2008 a março de 2010. A fazenda possuía 900 vacas em lactação de composição genética de $3 / 4$ a 31/32 Holandês Zebu, produzia em torno de 20.000 litros/ha/ano em sistema de semi-confinamento, com média total de $5.400 \mathrm{~kg}$ em 305 dias de lactação.

As informações obtidas no banco de dados foram: data de parição (chuvas - novembro a março, seco - abril a outubro), ordem de parição, inseminações, touros, diagnóstico de gestação presença da placenta 24 horas após o parto, tratamentos de desordens reprodutivas e pesagem quinzenal de leite. Na data de retirada dos dados do sistema, os animais entraram no cálculo de incidência, mas não entraram na avaliação de desempenho reprodutivo.

Para animais com período de lactação maior que 270 ou menor ou igual a 305 dias, utilizou-se a produção total na lactação, e para os animais com lactação acima de 305 dias, a produção foi corrigida para 305 dias segundo software de controle de rebanho utilizado na propriedade (IDEAGRI, Belo Horizonte, MG, Brasil). Para a comparação de pico de produção foi utilizado o maior valor da produção de leite na lactação. Para a comparação do efeito de curto prazo na produção de leite foram utilizados os dados de controle leiteiro até os 100 dias da lactação.

Animais descartados por motivos não relacionados à reprodução, sem dados encerrados de lactação ou períodos curtos de lactação sem informação sobre retenção de placenta ou não, na data de retirada dos dados do sistema, entraram no cálculo de incidência, mas não entraram na avaliação de desempenho produtivo.

Cálculo do impacto econômico. No estudo foram considerados os custos diretos (tratamento, mão de obra, redução da produção de leite e descarte de leite durante o período de tratamento) e indiretos (aumento do período de serviço, aumento do número de doses de sêmen e aumento do risco de descarte) para a retenção de placenta de acordo com metodologia de Kossaibati \& Esslemont (1997).

Os valores médios relacionados ao preço do tratamento, remuneração de mão de obra, preço anual do litro de leite pago pela empresa captadora, volume de leite descartado por dia de período de carência, preço da novilha ao parto, preço da vaca descartada involuntariamente, custo do dia de aluguel de pastagem para um animal e preço da dose de sêmen foram obtidos junto ao veterinário responsável pela propriedade com referência ao ano de 2009.

Levantamento dos custos diretos. Tratamento: a fazenda utilizou o protocolo padrão para todos os animais que apresentaram retenção de placenta por período superior a 24 horas. Esse tratamento consistia de aplicações de uma dose única de $2 \mathrm{ml}$ de Cipionato de estradiol $(2 \mathrm{mg} / \mathrm{ml}$ ) e $150 \mathrm{ml}$ (três doses de $50 \mathrm{ml}$ a cada 24 horas) de Oxitetraciclina $200 \mathrm{mg} / \mathrm{ml}$, como adoção curativa. E como suporte ao tratamento, era fornecido solução para hidratação pós-parto constituída por $300 \mathrm{ml}$ de propilenoglicol, $240 \mathrm{~g}$ de cloreto de sódio, $30 \mathrm{~g}$ de cloreto de potássio, $30 \mathrm{~g}$ de cloreto de cálcio e $30 \mathrm{~L} \mathrm{de}$ água. Não foram calculados os gastos com outros protocolos, por serem adotados igualmente a todos os grupos como, por exemplo, 
vermifugação e vacinações. A metodologia de cálculo do custo do tratamento por caso de retenção de placenta foi:

$$
C_{T R}=\left(P_{C} x N_{C}\right)+\left(P_{S} x N_{S}\right)
$$

$\mathrm{C}_{\mathrm{TR}}=$ Custo total do tratamento por caso de retenção de placenta em dólar (US\$),

$\mathrm{P}_{\mathrm{C}}=$ Preço da medicação curativa por unidade (ml, frasco ou litro) em dólar (US\$),

$\mathrm{N}_{\mathrm{C}}=$ Número de unidades (ml, frasco ou litro) utilizadas na medicação curativa,

$\mathrm{P}_{\mathrm{S}}=$ Preço da medicação suporte por unidade (ml, frasco ou litro) em dólar (US\$),

$\mathrm{N}_{\mathrm{S}}=$ Número de unidades ( $\mathrm{ml}$, frasco ou litro) utilizadas na medicação suporte.

Mão de obra: somente um funcionário foi designado para realização do tratamento das vacas com retenção de placenta. 0 custo com assistência veterinária não foi computado. A metodologia de cálculo do custo da mão de obra por ocorrência da retenção de placenta foi:

$$
C_{M O}=R /(C \times T)
$$

$\mathrm{C}_{\mathrm{MO}}=$ Custo total da mão de obra por caso de retenção de placenta em dólar (US\$),

$\mathrm{R}=$ Custo total mensal do funcionário composto por salário mínimo (US\$ 451,4), encargos (US\$ 84,4), férias (US\$12,5) e $13^{\circ}$ salário (US $\$ 36,6$ ),

C = Carga horária de trabalho mensal em horas (180h),

$\mathrm{T}=$ Tempo gasto em cada caso de retenção de placenta em horas $(0,5 \mathrm{~h})$.

Levou-se em consideração o custo do tempo gasto pelo funcionário em cada caso de retenção de placenta em relação do custo total do funcionário para a fazenda.

Redução da produção de leite: Apesar da redução da produção de leite impactar diretamente na redução da receita e não propriamente em aumento do custo, foi utilizada a metodologia de cálculo de Kossaibati \& Esslemont (1997) considerando o item como custo. A metodologia de cálculo do custo médio da redução da produção de leite por caso de retenção de placenta foi:

$$
C_{P L}=V_{L} x P_{L}
$$

$\mathrm{C}_{\mathrm{PL}}=$ Custo médio da redução da produção de leite por caso de retenção de placenta em dólar (US\$),

$\mathrm{V}_{\mathrm{L}}=$ Volume total médio de leite produzido a menos pelos animais com retenção de placenta em litros (L),

$\mathrm{P}_{\mathrm{L}}=$ Preço médio anual do litro de leite pago pela empresa captadora em dólar por litro (US\$/L).

Para avaliar a diferença de produção de leite entre os grupos com e sem retenção de placenta foi realizada análise de variância não paramétrica utilizando-se o teste estatístico de Kruskall Wallis e o teste de Mann-Whitney $(\mathrm{P}<0,05)$ (Nobre et al. 2012). 0 custo da redução da produção de leite foi considerado nos cálculos quando ocorreu diferença $(\mathrm{p}<0,05)$ de produção entre os grupos. Como não houve diferença $(\mathrm{p}<0,05)$ na produção de leite de vacas com e sem retenção de placenta nas diferentes épocas de parição (Nobre et al. 2012), esse item não foi contabilizado no cálculo do impacto econômico.

Descarte do leite durante o tratamento: o descarte do leite foi necessário no caso do tratamento com o medicamento Oxitetraciclina que exige período de carência para utilização do leite para consumo humano. 0 tempo de carência de sete dias foi definido por recomendação da bula da Oxitetraciclina ${ }^{\circledR}$ L.A. da empresa Bayer. Apesar do descarte do leite impactar diretamente na redução da receita e não propriamente em aumento do custo, utilizou-se no trabalho a metodologia de cálculo de Kossaibati \& Esslemont (1997) considerando o item como custo. A metodologia de cálculo do custo de descarte do leite por caso de retenção de placenta foi:

$$
C_{D L}=V_{L} x P_{L} x T
$$

$\mathrm{C}_{\mathrm{DL}}=$ Custo médio do descarte de leite por caso de retenção de placenta em dólar,

$\mathrm{V}_{\mathrm{L}}=$ Volume médio de leite descartado por dia no período de carência pelos animais com retenção de placenta em litros por dia (L/dia),

$\mathrm{P}_{\mathrm{L}}=$ Preço médio anual do litro de leite pago pela empresa captadora em dólar por litro (US\$/L),

$\mathrm{T}=$ Tempo de carência do produto utilizado no tratamento em dias.

Para a correção do custo total, os cálculos foram realizados de acordo com inflação acumulada no período de 2009 pelo IGP-M, fornecido pela Fundação Getúlio Vargas.

Levantamento dos custos indiretos. Aumento do período de serviço: de acordo com metodologia usada por Souza (2005), para fins de cálculo, os animais tiveram o período de lactação ajustado em até 305 dias, de forma que os gastos adicionais com o período de serviço foram calculados como o animal estando no período seco. O custo com a mão de obra do tratador responsável pelo manejo do lote de animais no período seco não foi computado. 0 custo médio de aluguel da pastagem (inclusa alimentação) utilizado foi de US\$0,38/animal/dia. A metodologia de cálculo do custo total do aumento do período de serviço causado por ocorrência da retenção de placenta foi:

$$
C_{P S}=C_{A} x\left(\mathrm{PS}_{\mathrm{C}}-\mathrm{PS}_{\mathrm{S}}\right)
$$

$\mathrm{C}_{\mathrm{PS}}=$ Custo total do aumento do período de serviço causado por ocorrência da retenção de placenta em dólar (US\$),

$\mathrm{C}_{\mathrm{A}}=$ Custo por animal por dia de aluguel de pastagem (inclusa alimentação) em dólar (US\$),

$\mathrm{PS}_{\mathrm{C}}=$ Período de serviço médio de vacas com retenção de placenta em dias,

$\mathrm{PS}_{\mathrm{S}}=$ Período de serviço médio de vacas sem retenção de placenta em dias.

Para avaliar a diferença de período de serviço entre os grupos com e sem retenção de placenta foi realizada análise de variância não paramétrica utilizando-se o teste estatístico de Kruskall Wallis e o teste de Mann-Whitney $(\mathrm{P}<0,05)$ (Nobre et al. 2012). 0 custo do aumento do período de serviço somente foi considerado nos cálculos quando ocorreu diferença $(\mathrm{p}<0,05)$ entre os grupos avaliados.

Aumento de número de doses de sêmen por gestação: De acordo com metodologia usada por Souza (2005), considerou-se somente a dose de sêmen no custo adicional referente ao aumento de serviços por gestação. 0 custo com a mão de obra por inseminação adicional não foi computado. A metodologia de cálculo do custo do aumento de serviços por gestação causada por ocorrência da retenção de placenta foi:

$$
C_{S C}=P_{d} x\left(N_{C}-N_{S}\right)
$$

$\mathrm{C}_{\mathrm{SC}}=$ Custo do aumento do número de doses por ocorrência da retenção de placenta em dólar (US\$),

$\mathrm{P}_{\mathrm{d}}=$ Preço médio da dose de sêmen em dólar (US\$14,3),

$\mathrm{N}_{\mathrm{C}}=$ Número médio de doses de sêmen utilizado em vacas com retenção de placenta,

$\mathrm{N}_{\mathrm{S}}=$ Número médio de doses de sêmen utilizado em vacas sem retenção de placenta. 
O custo do aumento do número de doses de sêmen por gestação somente foi considerado nos cálculos quando ocorreu diferença $(\mathrm{p}<0,05)$ em análise estatística previamente adotada entre os animais que tiveram ou não retenção de placenta (Nobre et al. 2012).

Aumento do risco de descarte: A metodologia de cálculo do aumento do risco de descarte causado por ocorrência da retenção de placenta foi baseada no valor de reposição do animal do rebanho de acordo com a seguinte fórmula:

$$
C_{D V}=T_{D} x N x(P c-P i)
$$

$\mathrm{C}_{\mathrm{DV}}=$ Custo do descarte por ocorrência da retenção de placenta em dólar (US\$),

$\mathrm{T}_{\mathrm{D}}=$ Taxa média de descarte devido à retenção de placenta em percentual (2\%),

$\mathrm{N}$ = Número de casos de retenção de placenta no ano (105),

Pc = Preço médio da novilha ao parto em dólar (US\$914,3),

$\mathrm{P}_{\mathrm{i}}=$ Preço médio da vaca descartada involuntariamente em dólar (US\$428,6).

\section{RESULTADOS E DISCUSSÃo}

\section{Levantamento dos custos diretos}

A incidência de retenção de placenta foi de $12,8 \%$, entre 2008 e 2010 (Nobre et al. 2012). Wilson et al. (2004), relataram incidência de retenção de placenta de 7,2\% em primíparas e $12,2 \%$ em multíparas, Sartori et al. (2014) relataram incidência de $14,7 \%$ no inverno e 14,8\% no verão, englobando primíparas e multíparas. Apesar das diferenças nas condições de cada estudo as incidências foram próximas, o que demonstra que os dados utilizados no presente trabalho, para fazer a estimativa de perdas econômicas devido a essa doença, condizem com a realidade encontrada em fazendas leiteiras.

O valor total do tratamento curativo e suporte por ocorrência da retenção de placenta, de acordo com a equação 1, foi de US\$13,3 (Quadro 1). 0 preço da mão de obra, de acordo com a equação 2 , foi caso de retenção de placenta foi de US\$1,6.

As perdas econômicas com descarte de leite devido ao tratamento com Oxitetraciclina foi de US\$37,8 (Equação 3 e 4), considerando volume de 12 litros de descarte, sendo o litro pago ao produtor no valor de US\$0,45, por sete dias de descarte.

\section{Levantamento dos custos indiretos}

0 custo total do aumento do período de serviço (Equação 5) foi de US\$19,6 no período das chuvas e de US\$10,5 no período da seca (Quadro 2), sendo considerados os valores de 178 e 129 dias os períodos de serviço médios de vacas com retenção de placenta, respectivamente para os períodos de chuva e da seca, e de 127 e 102 os períodos de serviço médios das vacas sem retenção de placenta, respectivamente para os períodos de chuva e de seca.

0 aumento do número de doses de sêmen por gestação (Equação 6) na época das chuvas onerou US\$17,0 e na época da seca US\$8,6, sendo considerados os valores de 3,3 e 2,6 o número médio de doses de sêmen utilizado em vacas com retenção de placenta, respectivamente para os períodos de chuva e da seca, e de 2,1 e 2,0 o número médio de doses de sêmen utilizado em vacas sem retenção de placenta, respectivamente para os períodos de chuva e de seca.
Os resultados da perda econômica com correção por ocorrência de retenção de placenta foram de US\$51,8 para vacas primíparas e de US\$70,6 para as vacas multíparas no período da seca e de US\$87,9 no período das chuvas (Quadro 2). A simulação do impacto econômico total no ano de 2009 para a propriedade em estudo apresentou valor de US\$8.878,0 (Quadro 3).

As perdas econômicas relacionadas à retenção de placenta levantadas por Guard (1994) estão relacionadas com tratamento, redução na produção de leite e aumento do período de serviço, tendo sido estimado total de US\$285 por caso. Kossaibati \& Esslemont (1997) calcularam média de $£ 298,29$ por caso de retenção de placenta considerando tratamento, redução da produção de leite, aumento do intervalo de partos, aumento do risco de descarte e aumento do risco de descarga vulvar. Guard (1994) estimou o custo de US $\$ 315$ por caso de mastite e metrite nos EUA. Liang et al. (2017) utilizaram modelo de simulação stochastic e relataram custo de US\$150.41 por caso de retenção de placenta em primíparas e US\$313.49 por caso em multíparas. Nesse modelo, os autores consideraram os custos com assistência veterinária e tratamento, aumento do trabalho do produtor, perda de produção de leite e dias em aberto. No presente estudo não foram considerados os custos com descarte ou morte dos animais, pois segundo Rajala-Schultz \& Gröhn (1998) a retenção de placenta não tem alto risco para descarte. Por outro lado, a retenção de placenta tem alta e positiva relação com a metrite. Considerando os custos combinados de retenção de placenta e metrite, Liang et al. (2017) relataram custo de US\$322 para primíparas e de US\$576, valores mais altos que o verificado no presente estudo, possivelmente pela inclusão dos custos com descarte e morte de animais. Esses custos refletem a soma das duas doenças separadas uma vez que o modelo proposto não considerou a associação entre as duas doenças.

Considerando as perdas e os custos com a retenção de placenta no presente trabalho, 0,34\% da produção de leite anual total da propriedade avaliada foi perdida para arcar com os custos dessa doença. Mudanças nos custos dos insumos, mão de obra e do preço pago por litro de leite causarão menor ou maior impacto nessas perdas. Além disso, alguns pontos podem ter influenciado o menor custo da retenção de placenta nesse estudo quando comparado com outros. Entre eles, a ausência de redução na produção de leite assim como a não contabilização de possíveis custos incorridos pelo aumento do risco de associação com outras doenças além de diferenças metodológicas no cálculo.

Considerando o número de partos ocorridos no período avaliado, assim como a taxa de incidência média do rebanho de $11,3 \%$ no ano de 2009 , conclui-se que a retenção de placenta custou US $\$ 8.878,0$ ou 19.666 litros de leite, representando impacto econômico negativo importante. Na simulação de cenários de redução e aumento da incidência na propriedade tendo como referência o ano de 2009 (Quadro 4), nos cenários pessimistas $(13,14$ e 15\%), verificou-se que pequenas variações em pontos percentuais acentuam o prejuízo relacionado a retenção de placenta chegando a US $\$ 2.574,6$ na taxa de $15 \%$ de incidência ao ano. Por outro lado, nos cenários otimistas ( 9 e 10\%), existe oportunidade de redução do prejuízo com essa afecção. 
Quadro 1. Custo total do tratamento curativo e suporte utilizado em cada animal apresentando retenção de placenta por período superior a 24 horas

\begin{tabular}{ccc}
\hline Item & Preço/Quantidade & Quantidade \\
US\$ & $2 \mathrm{ml}$ & Custo total \\
\hline Cipionato de estradiol $2 \mathrm{mg} / \mathrm{ml}$ & $5,3$ (frasco de $10 \mathrm{ml})$ & 1,0 \\
Oxitetraciclina $200 \mathrm{mg} / \mathrm{ml}$ & $3,1$ (frasco de $50 \mathrm{ml})$ & $150 \mathrm{ml}$ \\
Solução de hidratação & $2,8(30$ litros) & 2,4 \\
Total & & 13,3 \\
\hline
\end{tabular}

Fonte: dados da propriedade.

Quadro 2. Custo total por ocorrência de retenção de placenta em vacas primíparas e multíparas em diferentes épocas de parição

\begin{tabular}{|c|c|c|c|c|c|c|}
\hline \multirow{3}{*}{ Custos } & \multirow{2}{*}{\multicolumn{2}{|c|}{$\begin{array}{l}\text { Primíparas } \\
\text { Seca/Chuva }\end{array}$}} & \multicolumn{4}{|c|}{ Multíparas } \\
\hline & & & \multicolumn{2}{|c|}{ Seca } & \multicolumn{2}{|c|}{ Chuva } \\
\hline & US\$ & $\%$ & US\$ & $\%$ & US\$ & $\%$ \\
\hline \multicolumn{7}{|l|}{ Diretos } \\
\hline Tratamento & 13,2 & 25,1 & 13,2 & 18,4 & 13,2 & 14,7 \\
\hline Mão de obra & 1,6 & 3,1 & 1,6 & 2,3 & 1,6 & 1,8 \\
\hline Redução da produção de leite & - & - & - & - & - & - \\
\hline Descarte do leite & 37,3 & 71,8 & 37,3 & 52,8 & 37,3 & 42,4 \\
\hline Subtotal & 52,1 & 100,0 & 52,7 & 73,5 & 52,7 & 58,9 \\
\hline \multicolumn{7}{|c|}{ Indiretos } \\
\hline Aumento do período de serviço & - & - & 10,5 & 14,7 & 19,6 & 21,9 \\
\hline Aumento de número de doses por gestação & - & - & 8,6 & 11,8 & 17,1 & 19,2 \\
\hline Subtotal & - & - & 19,1 & 26,5 & 36,7 & 41,1 \\
\hline Total & 52,7 & 100,0 & 71,8 & 100,0 & 89,5 & 100,0 \\
\hline Total com correção* & 51,8 & - & 70,6 & - & 87,9 & - \\
\hline
\end{tabular}

* Correção de acordo com inflação acumulada no período de 2009 pelo IGP-M, fornecido pela Fundação Getúlio Vargas.

Quadro 3. Simulação do impacto econômico da retenção de placenta no ano de 2009 para a propriedade leiteira em estudo

\begin{tabular}{lcccc}
\hline \multicolumn{1}{c}{ Item } & $\mathrm{N}$ & Casos de retenção & Custo por caso US\$ & Custo total US\$ \\
\hline Primíparas (Seca/Chuva) & 305 & 26 & 52,7 & $1.371,1$ \\
Multíparas (Seca) & 390 & 33 & 71,8 & $2.370,7$ \\
Multíparas (Chuva) & 233 & 46 & 89,5 & $4.116,1$ \\
Subtotal & & & $7.857,9$ \\
Aumento do descarte* & & & $1.020,0$ \\
Total & & & $8.878,0$
\end{tabular}

* Cálculo do aumento do risco de descarte (Equação 7).

Quadro 4. Simulação de seis cenários de incidência $(8 \%, 9 \%, 10 \%, 13 \%, 14 \%$ e 15\%) de retenção de placenta no rebanho de 2009 e do subsequente impacto econômico para a propriedade leiteira em estudo

\begin{tabular}{|c|c|c|c|c|c|c|c|c|}
\hline \multirow{2}{*}{ Item } & \multirow{2}{*}{$\mathrm{N}$} & \multirow{2}{*}{$\begin{array}{l}\text { Custo por } \\
\text { caso (US \$) }\end{array}$} & \multicolumn{6}{|c|}{ Cenário de incidência de retenção de placenta } \\
\hline & & & $8 \%$ & $9 \%$ & $10 \%$ & $13 \%$ & $14 \%$ & $15 \%$ \\
\hline Primíparas (Seca/Chuva, US\$) & 305 & 52,74 & 973,5 & $1.096,9$ & $1.212,9$ & $1.535,7$ & $1.645,4$ & $1.768,8$ \\
\hline Multíparas (Seca; US\$) & 390 & 71,84 & $1.683,2$ & $1.896,6$ & $2.083,3$ & 2.655 .2 & $2.844,8$ & $3.058,2$ \\
\hline Multíparas (Chuva; US\$) & 233 & 89,48 & $2.922,4$ & $3.292,8$ & 3.579 & $4.610,0$ & $4.939,3$ & $5.309,7$ \\
\hline Subtotal (US\$) & & & $5.579,1$ & $6.286,4$ & $6.875,5$ & $8.800,9$ & $9.429,5$ & $10.136,8$ \\
\hline Aumento do descarte (US\$) & & & 724,2 & 816,0 & 893,7 & $1.142,4$ & $1.224,0$ & $1.315,8$ \\
\hline Total (US\$) & & & $6.303,3$ & $7.102,4$ & $7.769,2$ & $9.943,3$ & $10.653,5$ & $11.452,6$ \\
\hline Em relação ao custo total de 20 & $\$)^{*}$ & & $-2.574,6$ & $-1.775,6$ & $-1.108,7$ & $+1.065,3$ & $+1.775,6$ & $+2.574,6$ \\
\hline
\end{tabular}




\section{CONCLUSÕES}

A ocorrência de retenção de placenta em fazendas leiteiras é um evento comum, mas poucos levantamentos de incidência e custos dessa doença em propriedades do Brasil são encontrados na literatura.

Na propriedade avaliada a incidência desse distúrbio foi de $12,8 \%$ no período avaliado e custou para a propriedade US $\$ 8.878,0$ ou 19.666 litros de leite no período avaliado. Esse cenário aponta para impacto econômico significativo dessa afecção no fluxo de caixa de fazendas leiteiras sendo importante a realização de novas estimativas de custos em diferentes cenários econômicos assim como a conscientização dos produtores sobre a importância de implantar medidas preventivas para a retenção de placenta como forma de minimizar as perdas por essa afecção.

\section{REFERÊNCIAS}

Beagley J.C., Whitman K.J., Baptiste K.E. \& Scherzer J. 2010. Physiology and treatment of retained fetal membranes in cattle. J. Vet. Intern. Med. 24(2):261-268. http://dx.doi.org/10.1111/j.1939-1676.2010.0473.x. PMid:20136715.

Dijkhuizen A.A. \& Morris R.S. 1996. Animal Health Economics: principles and applications. Post Graduation Foundation in Veterinary Science, Sydney. 315p.

Farzaneh N., Mohri M., Jafari A.M., Honarmand K. \& Mirshokraei P. 2006. Peripartal serum biochemical, haematological and hormonal changes associated with retained placenta in dairy cows. Comp. Clin. Pathol. 15(1):27-30. http://dx.doi.org/10.1007/s00580-006-0605-7.

Fourichon C., Seegers H., Bareille N. \& Beaudeau F. 1999. Effect of disease on milk production in the dairy cow: a review. Prev. Vet. Med. 41(1):1-35. http://dx.doi.org/10.1016/S0167-5877(99)00035-5. PMid:10416197.

Guard C.L. 1994. Costs of clinical disease in dairy cows. Proc. Annu. Cornell Conf. Vet., Ithaca, NY.

Han I.K. \& Kim I.H. 2005. Risk factors for retained placenta and the effect of retained placenta on the occurrence of postpartum diseases and subsequent reproductive performance in dairy cows. J. Vet. Sci. 6(1):5359. PMid:15785124.
Kelton D.F., Lissemore K.D. \& Martin R.E. 1998. Recommendations for recording and calculating the incidence of selected clinical diseases of dairy cattle. J. Dairy Sci. 81(9):2502-2509. http://dx.doi.org/10.3168/ jds.S0022-0302(98)70142-0. PMid:9785242.

Kossaibati M.A. \& Esslemont R.J. 1997. The costs of production diseases in dairy herds in England. Vet. J. 154(1):41-51. http://dx.doi.org/10.1016/ S1090-0233(05)80007-3. PMid:9265852.

LeBlanc S.J., Lissemore K.D., Kelton D.F., Duffield T.F. \& Leslie K.E. 2006. Major advances in disease prevention in dairy cattle. J. Dairy Sci. 89(4):1267-1279. http://dx.doi.org/10.3168/jds.S0022-0302(06)72195-6. PMid:16537959.

Liang D., Arnold L.M., Stowe C.J., Harmon R.J. \& Bewley J.M. 2017. Estimating US dairy clinical disease costs with a stochastic simulation model. J. Dairy Sci. 100(2):1472-1486. http://dx.doi.org/10.3168/jds.2016-11565. PMid:28012631.

Meça K.K.O., Vasconcelos A.C. \& Moro L. 2006. Inibição de apoptose e retardo da maturação placentária: um provável mecanismo da retenção de placentária na brucelose bovina, revisão de literatura. Biosci. J. 22:163-174.

Nobre M.M., Coelho S.G., Haddad J.P.A., Campos E.F., Lana A.M.Q., Reis R.B. \& Saturnino H.M. 2012. Avaliação da incidência e fatores de risco da retenção de placenta em vacas mestiças leiteiras. Arq. Bras. Med. Vet. Zootec. 64(1):101-107. http://dx.doi.org/10.1590/S0102-09352012000100015.

Rajala P.J. \& Grohn Y.T. 1998. Effects of dystocia, retained placenta, and metritis on milk yield in dairy cows. J. Dairy Sci. 81(12):3172-3181. http://dx.doi. org/10.3168/jds.S0022-0302(98)75883-7. PMid:9891264.

Sartori R., Pontes G.C.S., Monteiro P.L.J., Nascimento A.B., Melo L.F. \& Wiltbank M.C. 2014. 105 retained fetal membranes: incidence and effect on milk production and reproductive performance in dairy cows. Reprod. Fertil. Dev. 26(1):166-167. http://dx.doi.org/10.1071/RDv26n1Ab105.

Souza R.C. 2005. Enfermidades podais em vacas leiteiras: eficiência e custos de tratamentos, efeitos na produção e reprodução, histopatologia e aspectos econômicos. Tese de Doutorado em Ciência Animal, Escola de Veterinária, Universidade Federal de Minas Gerais, Belo Horizonte. 92p.

Wilson D.J., González R.N., Hertl J., Schulte H.F., Bennett G.J., Schukken Y.H. \& Gröhn Y.T. 2004. Effect of clinical mastitis on the lactation curve: A mixed model estimation using daily milk weights. J. Dairy Sci. 87(7):2073-2084. http://dx.doi.org/10.3168/jds.S0022-0302(04)70025-9. PMid:15328219.

Zobel R. \& Tkalčić S. 2013. Efficacy of ozone and other treatment modalities for retained placenta in dairy cows. Reprod. Domest. Anim. 48(1):121-125. http://dx.doi.org/10.1111/j.1439-0531.2012.02041.x. PMid:22594457. 